\title{
Reibung - ein unterschätztes Phänomen in der Verpackungslogistik
}

\author{
DiPL.-ING. NORBERT BIERMANN \\ FACHGEBIET LOGISTIK, UNIVERSITÄT DORTMUND
}

Zusammenfassung

„Geiz ist geil!“ ist das Motto eines Handelshauses. Aber diese Philosophie erweist sich immer häufiger als ungeeignet, längerfristigen Erfolg zu sichern. Oftmals wird gerade aus diesem Grund wieder verstärkt auf Qualität geachtet, auf die Qualität von Produkten, auf die Qualität von Herstellungsprozessen, auf die Qualität von Logistikprozessen etc. Dieser Sinneswandel beeinflusst auch alle Verpackungsprozesse, da diese untrennbar mit der Sicherung der Produktqualität und der sicheren Abwicklung aller logistischen Prozesse verbunden ist. Neben der Forderung nach einem wirtschaftlichen Produktschutz als Kernaufgabe der Verpackung müssen jedoch auch zwingende Vorgaben - beispielsweise seitens des Gesetzgebers (z. B. im Lebensmittelbereich, in der Gefahrgutlogistik, im Straßenverkehrsrecht) beachtet werden. Das führt u. a. dazu, dass alle verpackten Güter so geschützt sein sollten, dass sie den Belastungen im Transportprozess, aber auch den Belastungen aufgrund von Ladungs- und Ladeeinheitensicherungsmaßnahmen standhalten können. Da sich jedoch nicht alle Ladegüter oder Packstücke beliebig für form- oder kraftschlüssige Sicherungsmaßnahmen eignen, sollte bei der Auslegung von Verpackungsmaßnahmen insbesondere der hilfreichen Wirkung von Reibungskräften zur Reduzierung zusätzlicher Sicherungsmaßnahmen Aufmerksamkeit gewidmet werden.

\begin{abstract}
„Geiz ist geil!“ is the motto of a famous German retailer, which means something like "stinginess is randy”. But this philosophy more and more proves itself to be inappropriate for having success in the long term. Because of this, it is increasingly looked after the quality of products, manufacturing processes, logistical processes and so on. This change of mind also influences all packaging processes, as these are intrinsically tied to the security of the product quality and the secure processing of all logistical processes. Besides the demand for an economical protection of products as a core function of the packing, mandatory guidelines - e.g. in the food industry, for dangerous goods or provisions of traffic law - have to be respected. This leads to the aim that all packaged goods should be secured in such a way, that they can withstand the stresses and strains during the transportation process as well as the stresses and strains caused by load and load unit securing measures. Because not all goods are equally suited for form-fit or force-fit securing measures, especially the helpful effect of friction forces for the reduction of additional securing measures should be considered when designing packaging techniques.
\end{abstract}

As a tool for increasing the friction forces, non-slip mats are preferred since many years, and especially in the youngest past innovation-savvy manufacturers are active on the market with high-value new developments featuring high coefficients of friction and good abrasion behaviour. But the usage of such slip-inhibiting materials for load securing imperatively requires reliable characteristic values for the quality rating of securing measures.

\section{Zentrale Verpackungsaufgaben}

Der Schutz von versendeten Gütern vor Transportschäden bei der Distribution vom Hersteller bis zum Empfänger sowie der Schutz von Verkehrsteilnehmern insbesondere im Straßengüterverkehr vor Gefahren durch transportierte Güter gehören zu den zentralen Aufgaben der Verpackungslogistik. Dies betrifft vor allem die Maßnahmen zur Sicherung von verladenen Gütern gemäß der Richtlinie VDI 2700 ff. und zur Sicherung versandfertiger Ladeeinheiten gemäß der Richtlinie VDI 3968 ff. Die Aufgabe des Produktschutzes unterliegt vor diesem Hintergrund allein der wirtschaftlichen Entscheidung des Produzenten und Verpackers, indem er die Aufwendungen für Verpackungsmaßnahmen zu dem jeweiligen Produktwert ins Verhältnis setzen kann. Die Aufgabe, unbeteiligte Verkehrsteilnehmer oder fremde Sachwerte vor Schäden durch das versendete Gut zu schützen, ist jedoch nicht primär eine kostenrelevante, sondern eine vom Gesetzgeber zwingend geforderte Sicherungsaufgabe. 
In der Vergangenheit sind Verpackungsmaßnahmen unter der Prämisse, den Produktschutz angemessen zu gewährleisten, oftmals als Reihenprozess erfolgt. Dabei werden beispielsweise die Produkte zunächst in eine Produktverpackung eingebracht, diese Verpackungseinheit wird anschließend durch ergänzende Polster- und Korrosionsschutzmaßnahmen geschützt, weiterhin können zusammenfassende Transport- oder Verkaufsverpackungen eingesetzt werden. Während kleinere Versandeinheiten oftmals mit Post- oder KEPDienstleistern in klassischen Transportverpackungen versendet werden, werden größere Mengen bevorzugt zu palettierten Ladeeinheiten zusammengestellt und gegen Auseinanderfallen und den Verlust einzelner Packstücke gesichert. Diese Ladeeinheiten werden ggf. wiederum auf Transportfahrzeuge verladen und dort gegen Verrutschen, Herabfallen etc. gesichert.

Es zeigt sich jedoch immer wieder, dass diese reihenorientierte Betrachtung der Verpackungslogistik nicht Ziel führend und Erfolg versprechend ist, da in der Regel die Wechselwirkungen zwischen den einzelnen Verpackungsteilprozessen nicht bedacht werden und da zudem oftmals auch unterschiedliche Unternehmen für die Realisierung der einzelnen Verpackungsteilprozesse zuständig sind. Aufgrund unterschiedlicher Verantwortungsbereiche unterbleiben oftmals die erforderlichen Abstimmungen zwischen den jeweiligen Prozessbeteiligten.

\section{Ganzheitliche Verpackungslogistik}

Die zeigt sich u. a. darin, dass eine Vielzahl von Normen und Richtlinien verfügbar ist, mit deren Hilfe die Eignung von Verpackungen vornehmlich für die Aufgabe des Produktschutzes und der Materialflussgängigkeit festgestellt werden kann. Weitere Verpackungsteilprozesse zur Sicherung von Ladeeinheiten (vgl. VDI 3968 ff.) und zur Ladungssicherung (vgl. VDI 2700 ff.) sind lediglich in den genannten VDI-Richtlinien sowie wenigen Prüfnormen, beispielsweise zur Zertifizierung von Zurrmitteln, erfasst.

Die Basis zur Bewertung dieser Transportsicherungsmaßnahmen beruhen auf gesetzlichen Regelungen wie beispielsweise der StVO, der StVZO, des HGB, verschiedener Normen und Unfallverhütungsvorschriften sowie der Richtlinien VDI 2700 ff. Hiernach ist es zwingend erforderlich, die Ladung, einzelne Ladegüter sowie einzelne Packstücke derart auf der Ladefläche zu fixieren, dass bei normalen Fahrzuständen keine unzulässigen Verschiebungen auftreten können.

In diesem Zusammenhang ist immer wieder festzustellen, dass Ladungs- und/oder Ladeeinheitensicherungsmaßnahmen nicht in angemessener Weise durchgeführt werden können, da die vorangegangenen Verpackungsmaßnahmen mangelhaft ausgeführt worden sind. Oftmals können sie die für die Ladeeinheiten- und Ladungssicherung erforderlichen Sicherungskräfte nicht unbeschadet kompensieren.

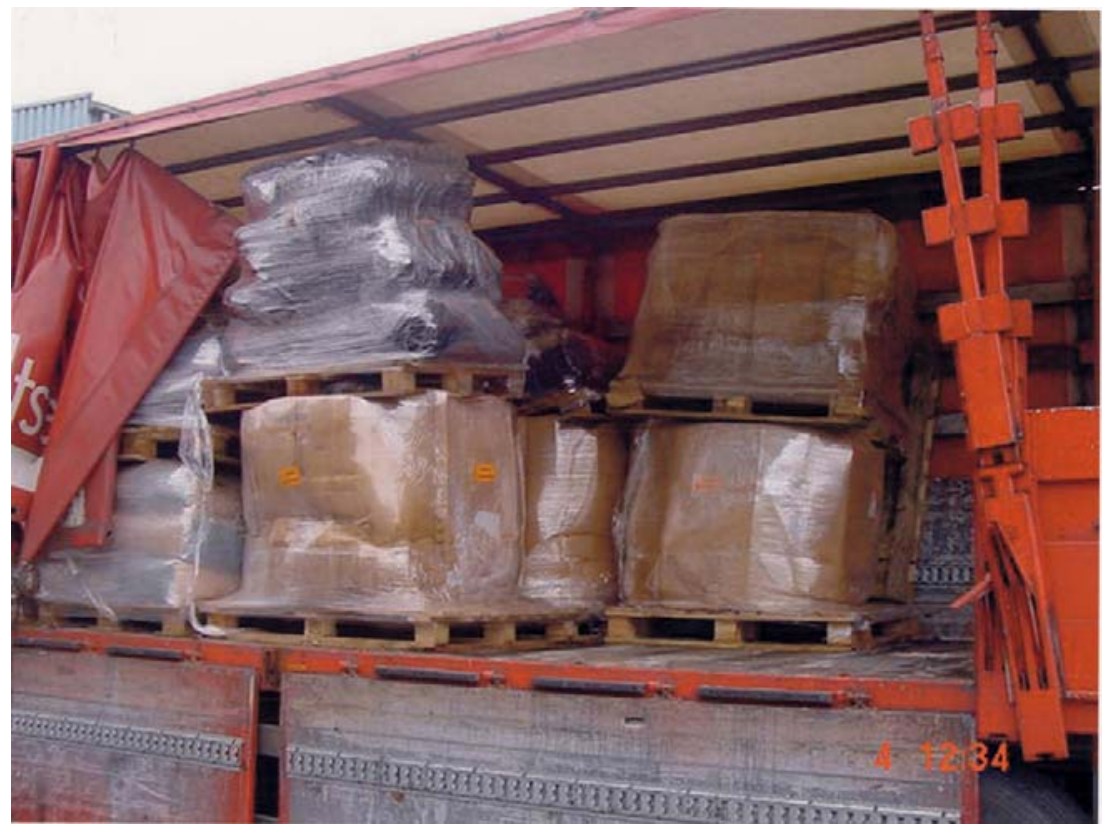

Abbildung 1: Beispiel für mangelhafte Ladungssicherung, Ladeeinheitensicherung und Verpackungsauslegung (Bildquelle: StAfA) 
Aus diesem Grund ist ein Umdenken erforderlich, welches den gesamten Verpackungsprozess mit allen Wechselwirkungen gleichsam als Regelkreis der Verpackungslogistik berücksichtigt und ggf. schon frühzeitig ausreichende Festigkeiten von Packstücken (z. B. zur Kompensation von Stapelkräften oder zur Aufnahme von Folienkräften) sicherstellt oder anderweitige, alternative Sicherungsmaßnahmen (z. B. Erhöhung von Reibungswiderständen) realisiert.

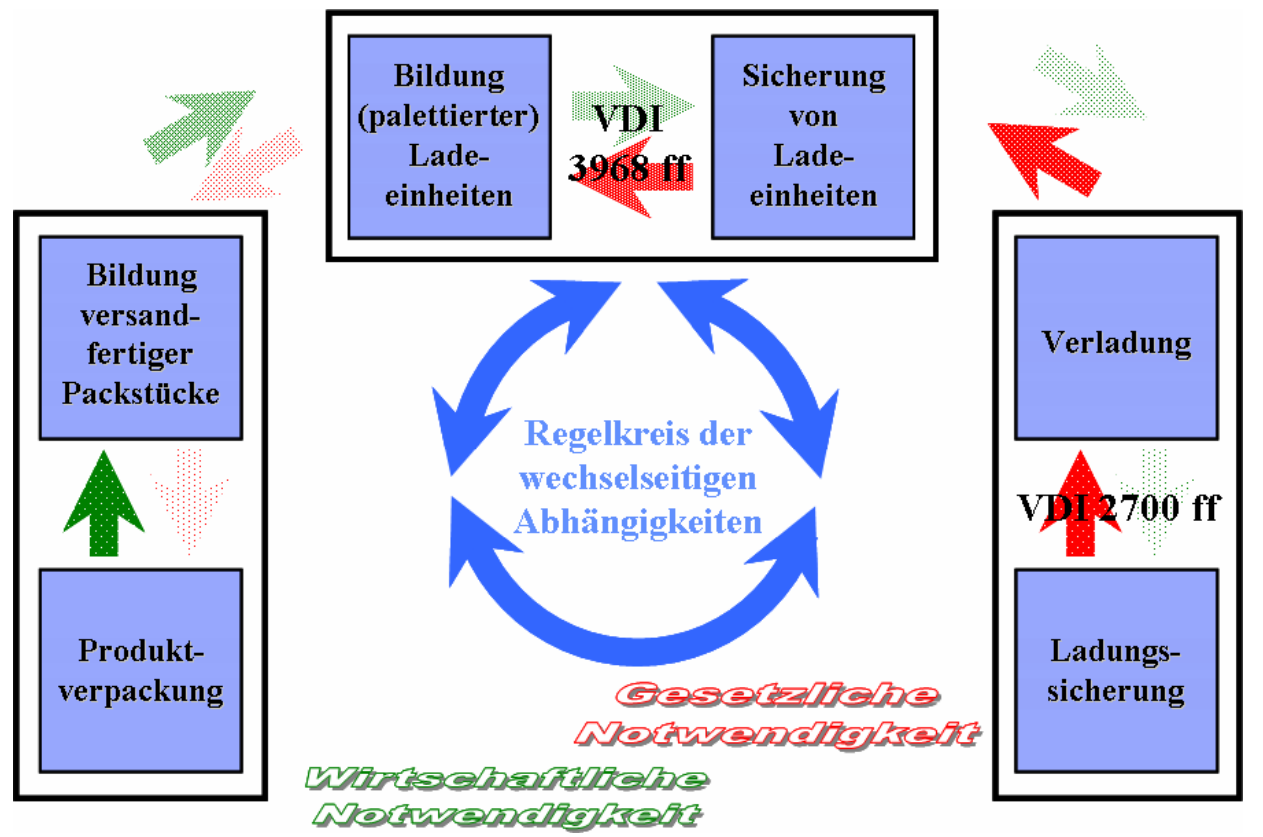

Abbildung 2: Regelkreis der Verpackungslogistik

\section{Kräfte zur Berechnung von Verpackungsmaßnahmen}

Die rechnerische Auslegung von Kräften zur Sicherung verladener Güter gemäß der Richtlinie VDI $2700 \mathrm{ff}$. kann vor diesem Hintergrund als genereller Berechnungsalgorithmus für viele Verpackungsauslegungen zugrunde gelegt werden.

So wirken bei Transportvorgängen auf einzelne Ladegüter sowie auf die gesamte Ladung unterschiedliche Kräfte, von denen für die Ladungssicherung insbesondere die Massen- und Reibungskräfte zu berücksichtigen sind. 


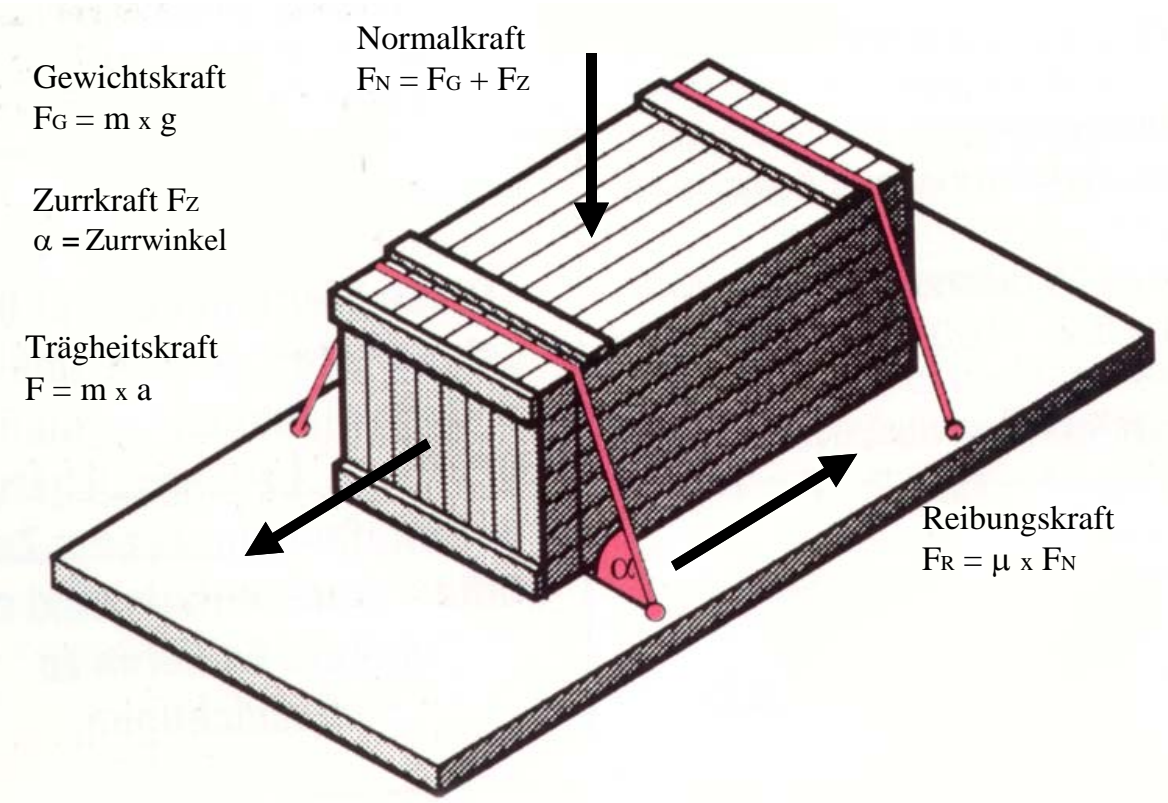

Abbildung 3: Ladungssicherungsrelevante Kräfte

Die Gewichtskraft $F_{G}$ eines Ladegutes bzw. der gesamten Ladung errechnet sich bekanntlich aus der jeweiligen Masse $m$ des einzelnen Ladegutes bzw. der Gesamtladung multipliziert mit der Erdbeschleunigung $g$ und ist zum Erdmittelpunkt gerichtet.

$$
F_{G}(N)=m(k g) \times g\left(m / s^{2}\right)
$$

Aufgrund der Massenträgheit hat jede Ladung das Bestreben, sich einer Änderung ihres Bewegungszustandes zu widersetzen. Einmal in Bewegung versetzt - Ladefläche und Ladegut bilden dabei ein relativ zueinander ruhendes System - ist die bewegte Einheit aufgrund ihrer Massenträgheit bestrebt, diese Bewegung mit unveränderter Geschwindigkeit und mit unveränderter Bewegungsrichtung beizubehalten. Diese der Beschleunigung beim Anfahren, bei Kurvenfahrt und beim Bremsen entgegen gerichtete Kraft wird als Massenkraft $\mathrm{F}$ bezeichnet. Die Ladung hat aufgrund ihrer Massenträgheit das Bestreben, sich relativ zur beschleunigten oder abgebremsten Ladefläche zu verschieben oder zu kippen.

Jedes Ladegut der Masse m erfährt bei einer solchen Änderung der konstanten, geradlinigen Bewegung des Systems Ladefläche/Ladegut eine Massen- oder Trägheitskraft F, die sich aus der Masse m des Ladegutes multipliziert mit der jeweiligen Beschleunigung a des Fahrzeugs (z. B. Beschleunigung bzw. Bremsen) errechnet und in bzw. entgegen zur Bewegungsrichtung des Fahrzeugs wirkt.

$$
\mathrm{F}(\mathrm{N})=\mathrm{m}(\mathrm{kg}) \times \mathrm{a}\left(\mathrm{m} / \mathrm{s}^{2}\right)
$$

Bei Kurvenfahrt errechnet sich die Massenkraft $\mathrm{F}$ aus der Masse $\mathrm{m}$ des Ladegutes multipliziert mit der Kurvenbeschleunigung $\mathrm{v}^{2} / \mathrm{r}$ des Fahrzeugs, wobei v die gefahrene Geschwindigkeit und $\mathrm{r}$ der Kurvenradius bezogen auf den Ladungsschwerpunkt S - ist. Diese Massenkraft ist bestrebt, die Ladung auf der Ladefläche in Richtung des Kurvenaußenrandes zu bewegen.

$$
\mathrm{F}(\mathrm{N})=\mathrm{m}(\mathrm{kg}) \times \mathrm{v}^{2}\left(\mathrm{~m}^{2} / \mathrm{s}^{2}\right) / \mathrm{r}(\mathrm{m})
$$

Dieser Massenkraft als Ursache vieler Transport- und Unfallschäden wirkt insbesondere die Reibungskraft $F_{R}$ entgegen, indem sie das Verrutschen einzelner Ladegüter oder Packstücke abbremst bzw. verhindert, und ist damit im Sinne einer Sicherungskraft wirksam. Die Reibungskraft $F_{R}$ errechnet nach derzeit verbreiteter Sichtweise sich aus der Gewichtskraft $\mathrm{F}_{\mathrm{G}}$ der Ladung multipliziert mit dem Reibbeiwert $\mu$.

$$
F_{R}(N)=\mu \times F_{G}(N)
$$

Reibbeiwerte, deren Höhe maßgeblichen Einfluss auf die weiteren Ladungssicherungsmaßnahmen haben, sind sehr stark abhängig von den Materialeigenschaften (z. B. der Oberflächenstruktur, der Festigkeit, der Härte und der Flächenpressung) und dem Zustand (z. B. trocken, nass und fettig) der sich berührenden Flächen der Reibungspartner, sowie von den dynamischen Belastungsprofilen (z. B. Schwingungen, Stöße) des Fahrbetriebs. 
Um die Dynamik von Transportvorgängen annähernd praxisgerecht zu erfassen, muss gemäß der Richtlinie VDI 2700 ff. für die Ladungssicherung nicht der Haftreibbeiwert sondern der Gleitreibbeiwert eingesetzt werden. Im Zweifelsfall ist hiernach für den Gleitreibbeiwert $\mu$ der niedrigste angegebene Wert einzusetzen, soweit nicht für den Einzelfall ermittelte und nachgewiesene Reibwerte für bestimmte Werkstoffpaarungen vorliegen.

Um der Ladung die Möglichkeit zu nehmen, relativ zur Ladefläche in Bewegung zu geraten, ist die Ladung zu sichern. Der Differenzbetrag zwischen Massenkraft F und wirksamer Reibungskraft $F_{R}$ wird schließlich als Sicherungskraft $F_{S}$ bezeichnet.

$$
F_{S}(N)=F(N)-F_{R}(N)
$$

Die Sicherungskraft $F_{S}$ ist die Kraft, die ergänzend zur Reibungskraft von den Sicherungsmitteln zur Sicherung der Ladegüter aufgenommen werden muss.

\section{Bedeutung der Reibung für die Verpackungslogistik}

Sobald rechnerisch oder ggf. prüftechnisch nachgewiesen ist, dass die ausgewählten Verpackungs- bzw. Sicherungsmaßnahmen zu dem geforderten Ergebnis, keine unzulässige Verschiebung aufgrund üblicher Transportbelastungen, führen, ist eine Bewertung der Wirtschaftlichkeit der einzelnen, geeigneten Varianten (z. B. Einsatz einer großen Anzahl von Zurrgurten vs. Einsatz von rutschhemmenden Materialien bei kraftschlüssiger Ladungssicherung) zulässig.

Ein Ansatz, zusätzliche Ladungssicherungsmaßnahmen zu minimieren oder auf die ladegutspezifischen Erfordernisse anzupassen (z. B. bei Verladung von nicht niederzurrfähigen Ladeeinheiten), liegt im Einsatz von rutschhemmenden Materialien zwischen der Ladefläche und dem Ladegut bzw. zwischen gestapelten Ladegütern.

Die Wirtschaftlichkeit derartiger Alternativen muss u. a. die Abschreibungskosten der verwendeten Sicherungsmittel (z. B. Zurrgurte, Antirutschmatten), die Personal-Zeit-Kosten für die Ausführung der Sicherungsmaßnahmen, die Inspektions- und Prüfkosten für die Sicherungsmittel und weitere kostenrelevante Aspekte (z. B. Blockade des Verladeplatzes während der Ladungssicherungsmaßnahme) berücksichtigen.

\section{Tabelle Einfachmethode Miederzunrem Anzahl der erforderlichen Zurrmittel}

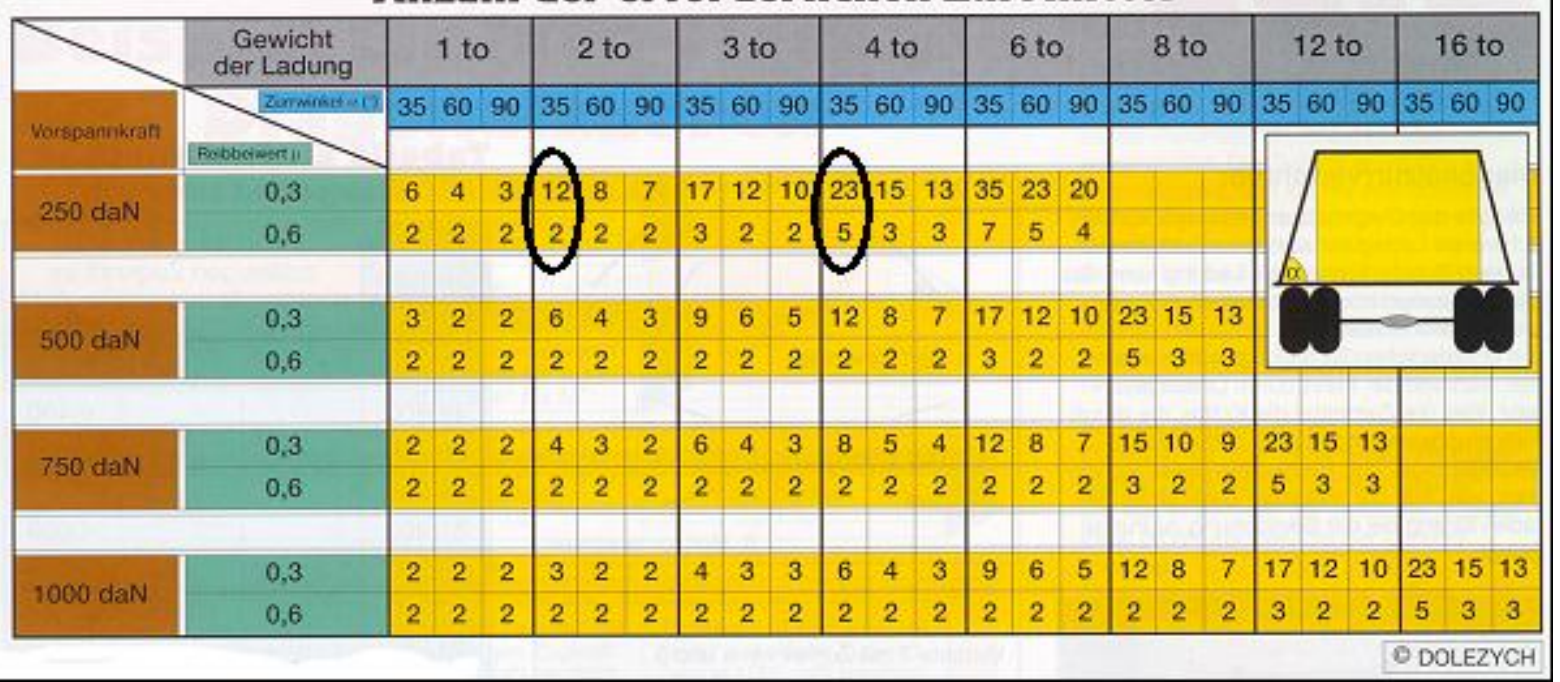

Abbildung 4: Ansatz zur Optimierung der Wirtschaftlichkeit von Ladungssicherungsmaßnahmen

Der Einsatz von rutschhemmenden Materialien zur Ladungssicherung setzt jedoch zwingend verlässliche Kennwerte zur Qualitätsbewertung der Sicherungsmittel voraus.

Die Notwendigkeit verlässlicher Materialkennwerte für die Berechnung von Reibungskräften ist in mehreren Forschungsprojekten des Fachgebiets Logistik der Universität Dortmund dargestellt worden. Im Rahmen dieser Forschungsprojekte AiF-FV-Nr.: 12292 und AiF-FV-Nr.: 14008 konnten die komplexen Wirkzusammenhänge der Reibung bei verladenen Gütern analysiert und verdeutlicht werden. Ergänzend zu diesen Forschungsergebnissen wird die Reibungsthematik derzeit in Zusammenarbeit mit Experten aus Industrie und Handel, von Kontrollbehörden sowie von Prüf- und Forschungseinrichtungen im Rahmen der VDI- 
Richtlinienarbeit (VDI 2700 Blatt 14 und 15) ausgearbeitet, sodass nach Fertigstellung der Richtlinien umfassende und praxisgerechte Hilfsmittel zur Berechnung der Reibungskräfte für die Ladungssicherung verfügbar sind.

\section{Arten unterschiedlicher ARM}

Als Mittel zur Erhöhung von Reibungskräften werden seit vielen Jahren Antirutschmatten bevorzugt, bei denen gerade in der jüngsten Vergangenheit innovationsfreudige Hersteller mit hochwertigen Neuentwicklungen, die sich u. a. durch hohe Gleitreibbeiwerte und gutes Verschleißverhalten auszeichnen, auf dem Markt aktiv sind. Eine Auswahl derartiger Antirutschmatten wird im Folgenden vorgestellt.

Überwiegend werden zur Zeit Antirutschmatten aus Gummigranulaten verwendet. Ausgangsrohstoffe dieser offen strukturierten ARM mit Hohlraumanteil sind Styrol-Butadien-Rubber (SBR) und Natural Rubber (NR) sowie Polyurethan (PUR) als Bindemittel. Die Rohstoffe werden üblicherweise mit Polyurethan-Bindemittel vermischt und zu Zylinderkörpern verpresst. Nach dem Aushärten werden davon Bahnen mit angepasster Dicke abgeschält.

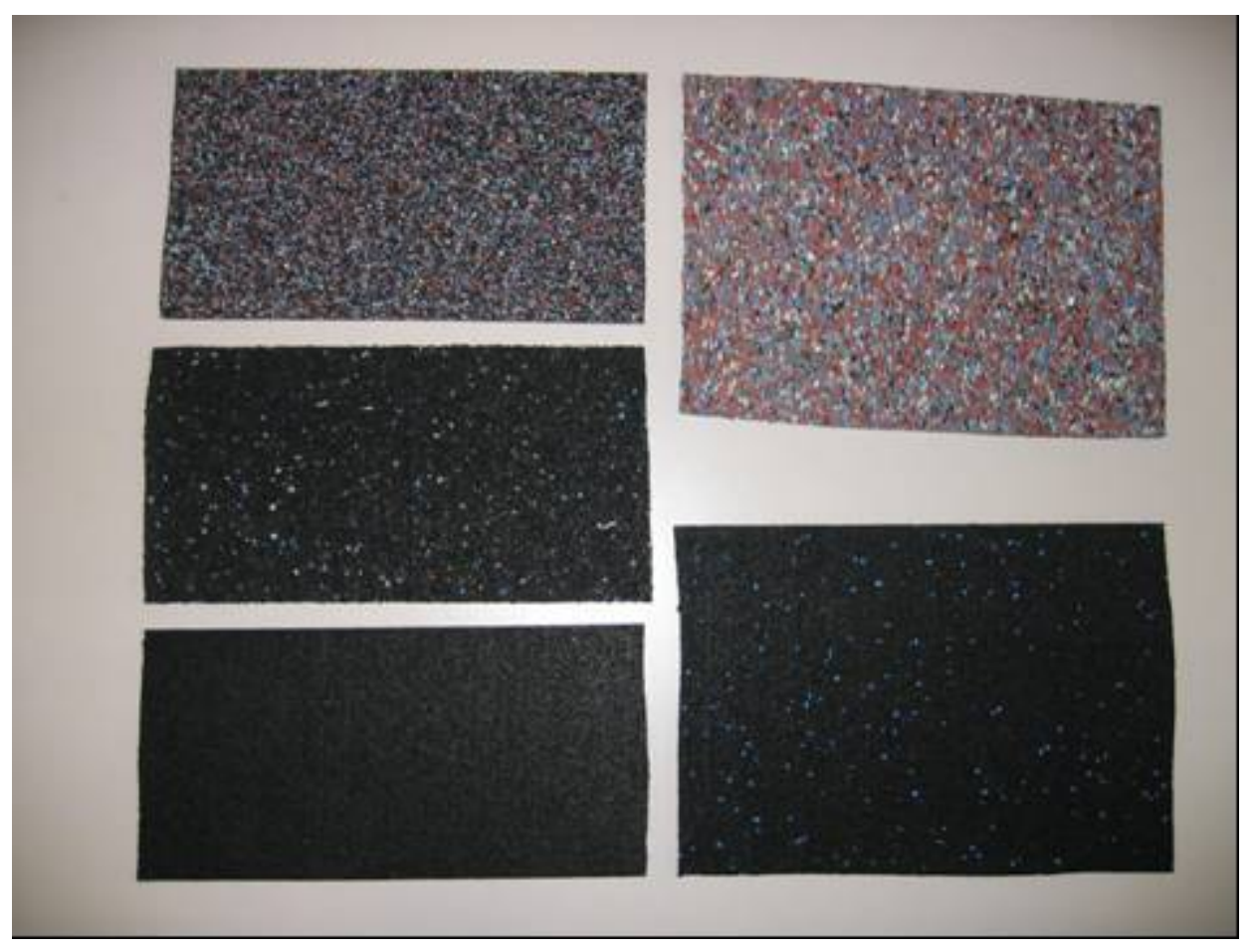

Abbildung 3: Beispiele ausgewählter Gummigranulat-ARM

Neben den Gummigranulat-ARM mit Hohlraumanteil werden auch solche verwendet, die massiv aus vulkanisiertem Gummi oder massiv aus kompakt gegossenem PUR bestehen. Ihre Oberfläche sollte strukturiert sein (z. B. Noppen, Rillen), um Schmutzpartikel und Feuchtigkeit aufnehmen zu können. Vulkanisierte MassivARM bestehen üblicherweise aus Neu-Gummi mit dem Füllstoff Reifengranulat aus SBR oder NR und werden weitgehend ohne Hohlraum-Anteil gefertigt. ARM aus Vollmaterial werden üblicherweise im Guss- oder Extrusionsverfahren hergestellt.

Massive ARM werden alternativ auch aus PUR mit Gewebe- oder Lochblecharmierung hergestellt, wobei jedoch keine Füllmaterialien verwendet werden. Polyurethan-Produkte werden hierbei aus 2-komponentigen Polyurethan-Rohstoffen hergestellt, die miteinander vermischt und bis zum Aushärten in Handformen gegossen werden. 


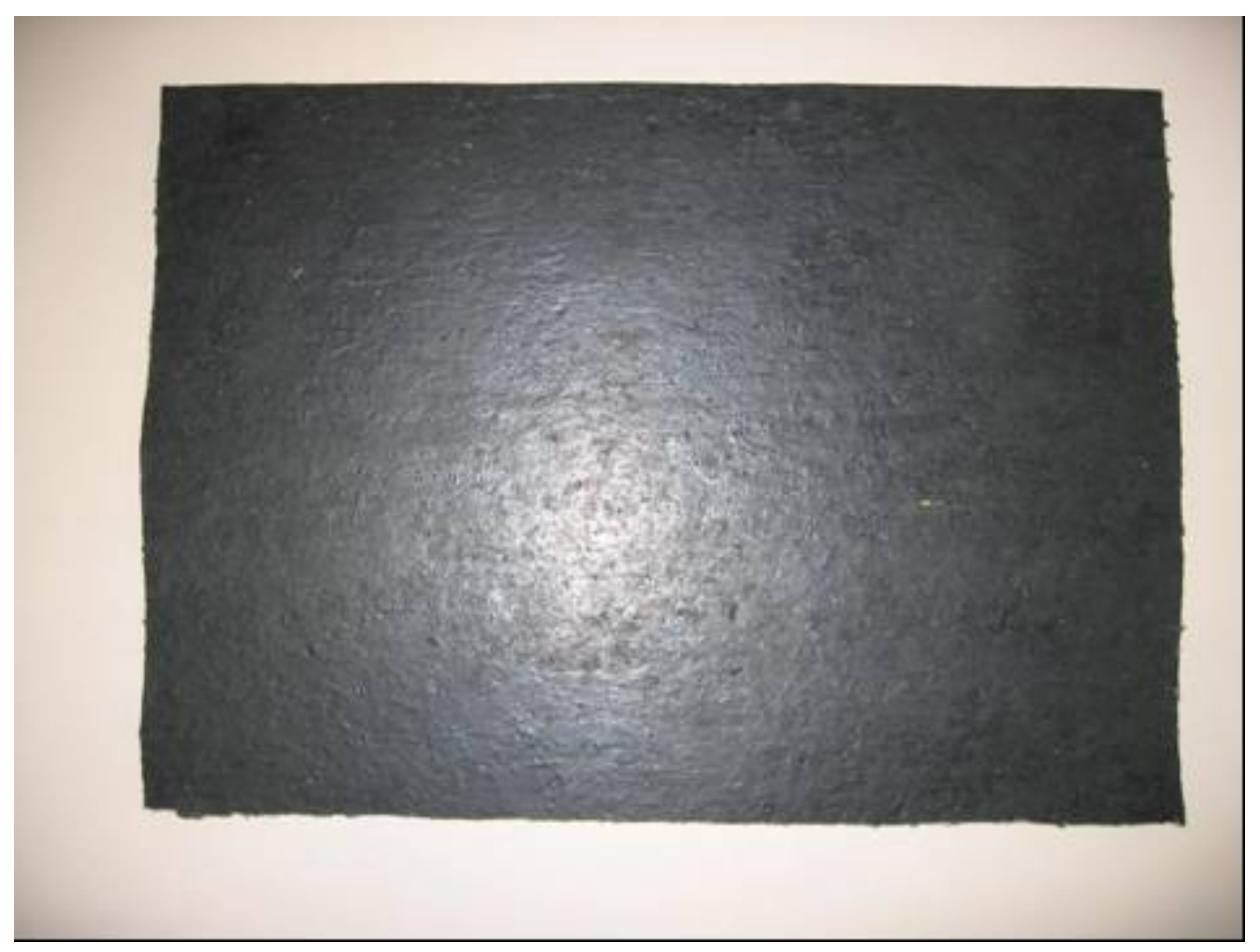

Abbildung 4: Beispiel einer Vollgummi-ARM

Das Trägermaterial von rutschhemmend mit Schaumstoff beschichteten Geweben besteht beispielsweise aus hochreißfesten Polyamid- und Polyesterfasern (PA und PE), die bei der Herstellung aus Kett- und Schussfäden mit einer genau festgelegten Garnstärke und Fadenzahl zu einem Gewirk verknüpft werden. Dies wird anschließend in die Beschichtungsanlage eingeführt, in der das Trägermaterial durch ein gesteuertes Tauchverfahren mit einer Schicht aus Polyvinylchlorid (PVC)-Schaumstoff gleichmäßig umhüllt wird. Im Anschluss wird das Produkt ausgehärtet und getrocknet.

Das Trägermaterial von rutschhemmend mit Schaumstoff beschichteten ARM besteht alternativ aus textilen Filzoder Vlies-Matten. Auch dieses Trägermaterial wird mit einer Schicht aus Polyvinylchlorid (PVC)-Schaumstoff versehen. 


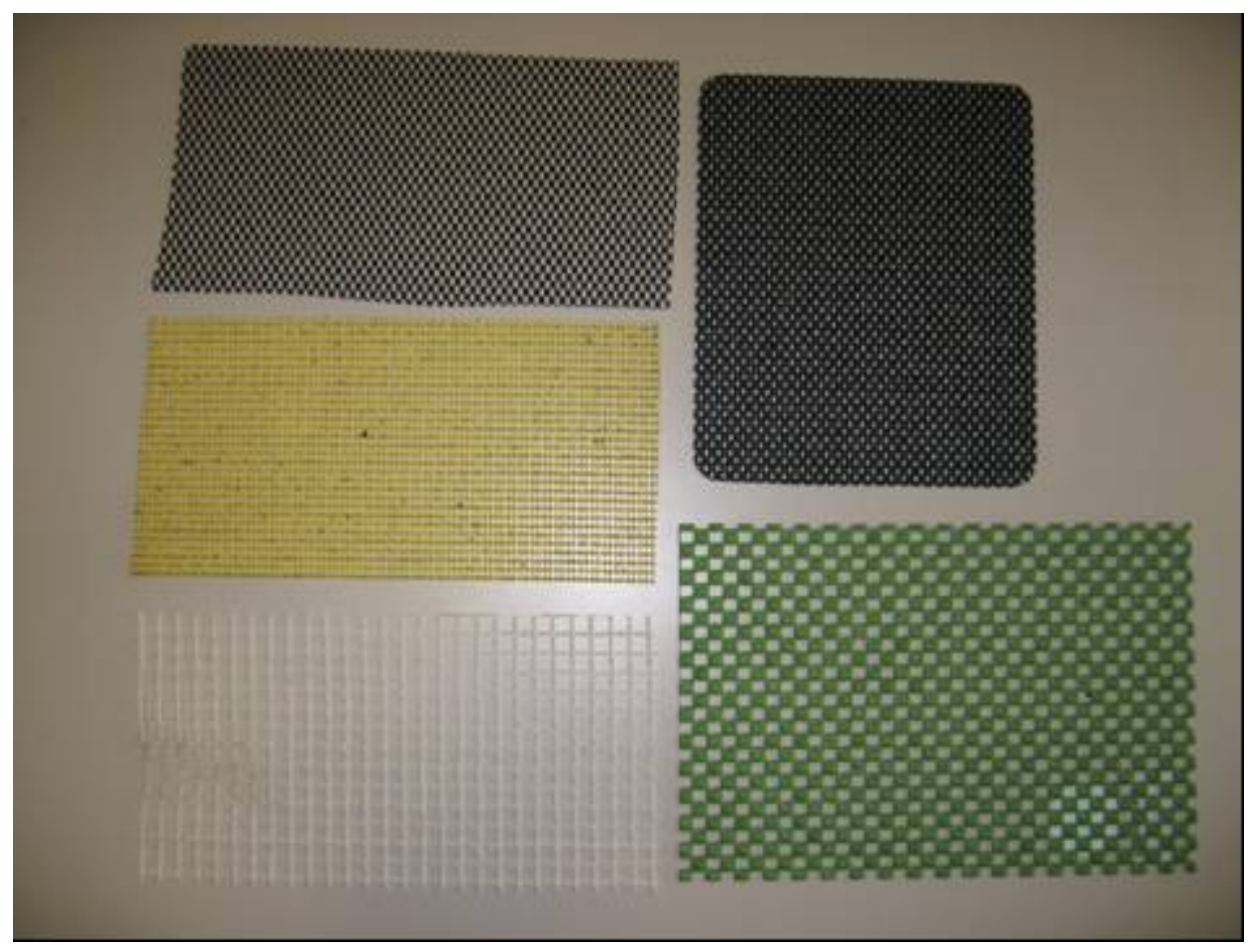

Abbildung 5: Beispiele ausgewählter ARM mit schaumstoffbeschichtetem, offenem Gewebe

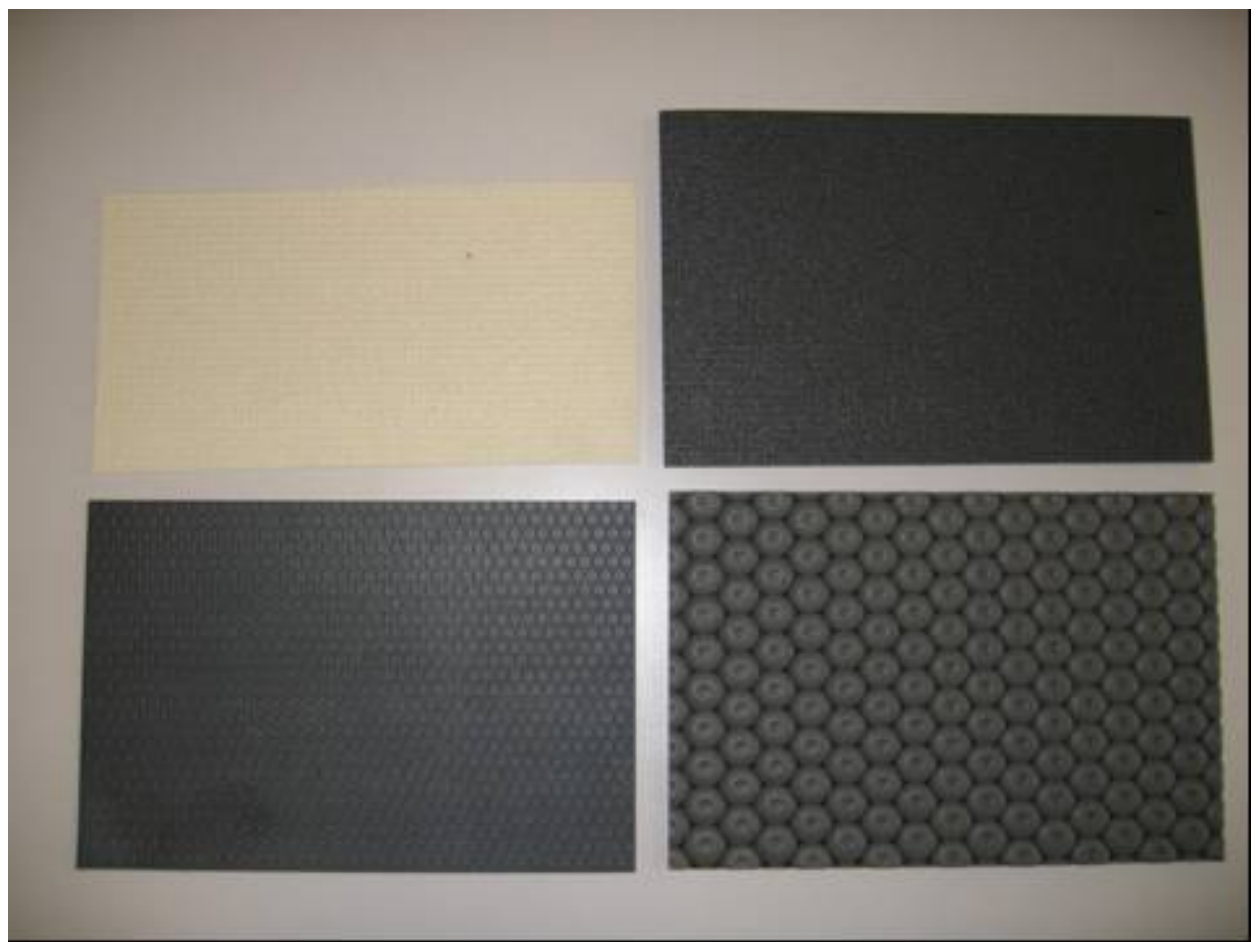

Abbildung 6: Beispiele ausgewählter ARM mit schaumstoffbeschichteten, geschlossenem Gewebe 
Als Grundmaterial von Filz-ARM dient oftmals reine Baumwolle, die mit einer adhäsiven (klebrigen) Komponente beschichtet oder getränkt wird.

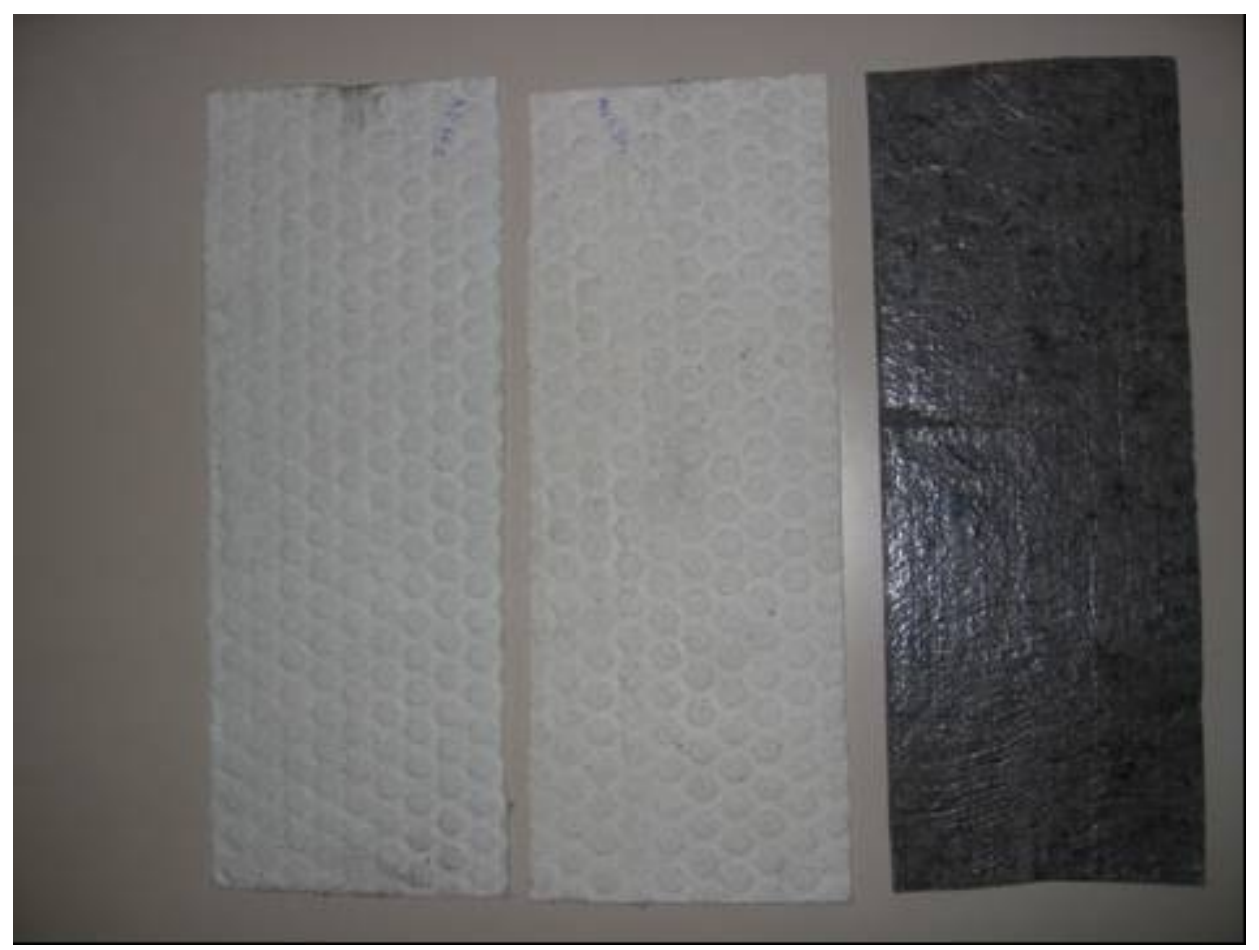

Abbildung 7: Beispiele von rutschhemmenden Filz-ARM

\section{Ladungssicherungsrelevante Eigenschaften von ARM}

Es ist naheliegend, dass diese Antirutschmatten - hier ist nur eine kleine Auswahl gängiger ARM vorgestellt worden - mit unterschiedlichen Materialeigenschaften hergestellt werden können, was die sachgerechte Auswahl einer geeigneten ARM für den Verlader oder den Transportunternehmer weiter erschwert. Aus diesem Grund werden hier einige wesentliche Auswahlkriterien vorgestellt und erläutert.

Als wichtigste Kennwerte von Antirutschmatten für die Ladungssicherung sind die Reibbeiwerte zu nennen. Die Haftreibbeiwerte sind jedoch aufgrund der VDI-Vorgaben nicht für Ladungssicherungsmaßnahmen relevant. Zudem können sie nicht immer ermittelt werden; insbesondere bei weichen, formveränderlichen Reibungspartnern - z. B. beim Einsatz weicher ARM oder nachgiebiger Ladegüter - erfolgt unter einer verschiebenden Belastung zunächst eine Verformung eines oder mehrere Reibungspartner ohne eine relative Verschiebung der jeweiligen Reibungspartner in den Kontaktflächen, die nahtlos in eine relative Verschiebung zwischen den Reibungspartnern übergeht.

Der für die Ladungssicherung maßgebliche Gleitreibbeiwert wird entscheidend beeinflusst durch die Anzahl und Größe der reibungswirksamen Kontaktflächen zwischen der Ladefläche bzw. der jeweiligen Unterlage - ARM Ladegut. Da Reibung als Widerstand gegen ein Verschieben von miteinander verzahnten Gegenständen betrachtet werden kann, sind die Gleitreibbeiwerte generell abhängig von allen Parametern, die die Wirksamkeit der Verzahnung beeinflussen (z. B. Rauigkeit der beteiligten Kontaktflächen, Weichheit und Nachgiebigkeit dieser Flächen, kontaktflächenbezogene Druckbelastung, Temperaturverhalten der Reibungspartner, Feuchtigkeit und Nässe zwischen den Reibungspartnern).

Darüber hinaus muss beim Einsatz von Antirutschmatten grundsätzlich beachtet werden, dass an keiner Stelle ein direkter Kontakt zwischen Ladegut und Ladefläche bzw. zwischen gestapelten Ladegütern besteht; andernfalls liegt eine undefinierte Mischreibung zwischen der jeweiligen Unterlage, der ARM und des Ladegutes vor und der Reibbeiwert der Materialpaarung Ladefläche bzw. Unterlage - ARM - Ladegut darf nicht zur Berechnung der Reibungskräfte herangezogen werden.

Mit der auf eine definierte Kontaktfläche bezogenen Druckbelastung (Flächenpressung) kann die Anzahl der reibungswirksamen Verzahnungen entscheidend beeinflusst werden. 
Aus diesem Grund muss der Einsatz von dünnen ARM dann abgelehnt werden, wenn aufgrund der Unebenheit der Ladefläche und/oder des Ladegutes die dazwischen liegende ARM nur an wenigen Kontaktpunkten belastet wird. Mit sinkender Anzahl wirksamer Kontaktpunkte sinken ebenfalls die wirksamen Reibungskräfte. Darüber hinaus besteht die Gefahr, dass die Antirutschmatten aufgrund einer Überlastung in den Druckbereichen geschädigt oder gar gänzlich zerstört werden können.

In letzter Konsequenz führen auch weitere Rahmenbedingungen auf der Ladefläche wie beispielsweise eine Materialversprödung bei niedrigen oder eine Materialerweichung bei höheren Temperaturen dazu, dass die Intensität der Verzahnung der Reibungspartner in den Kontaktflächen verändert wird, sodass sich auch diesbezüglich die heranzuziehenden Reibbeiwerte verändern.

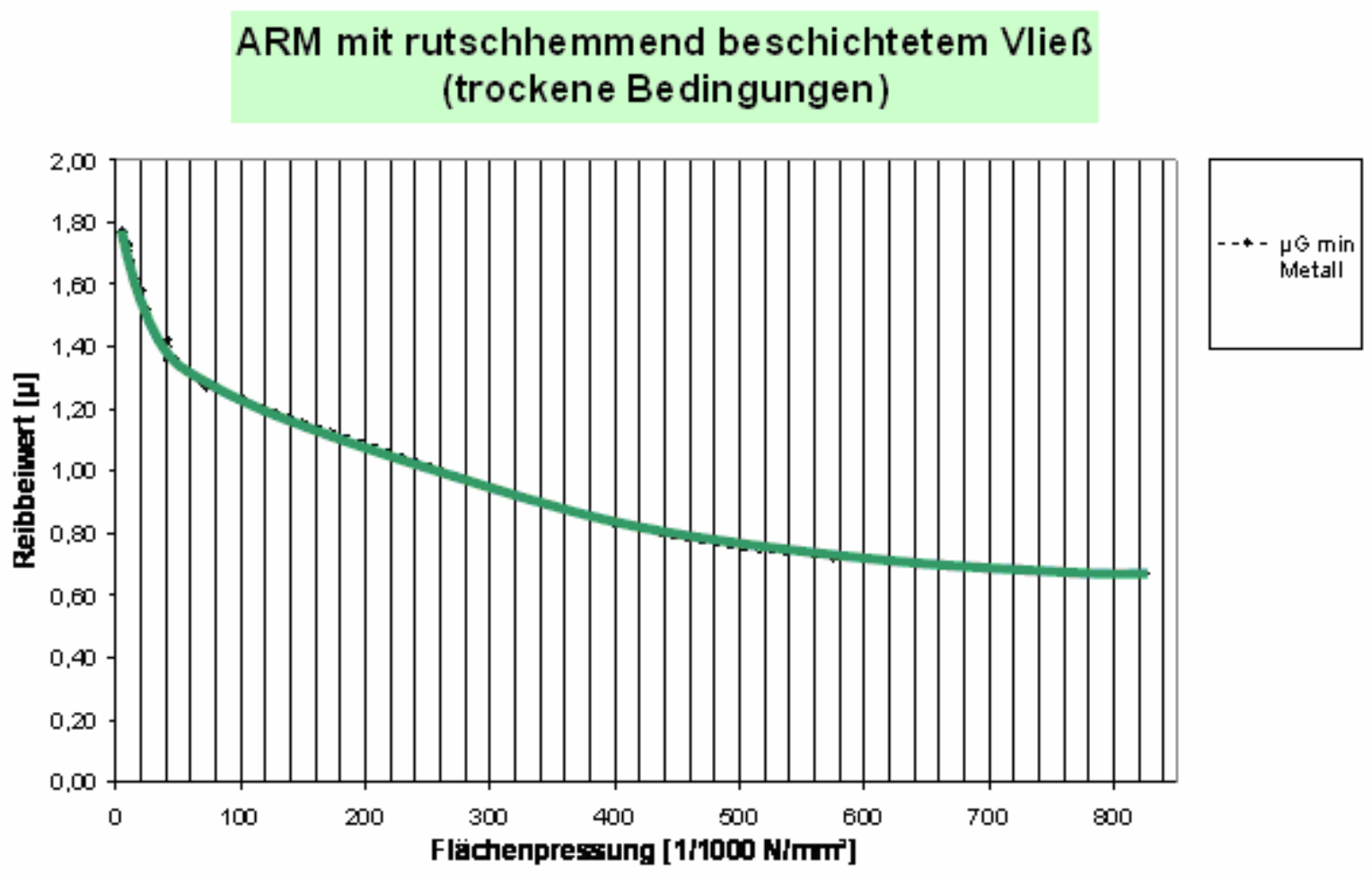

Abbildung 8: Diagramm der minimalen Gleitreibbeiwerte bei unterschiedlichen Flächenpressungen (Metall - ARM - Metall, trocken) 


\section{ARM mit rutschhemmendbeschichtetem Vließ} (feuchte Bedingungen)

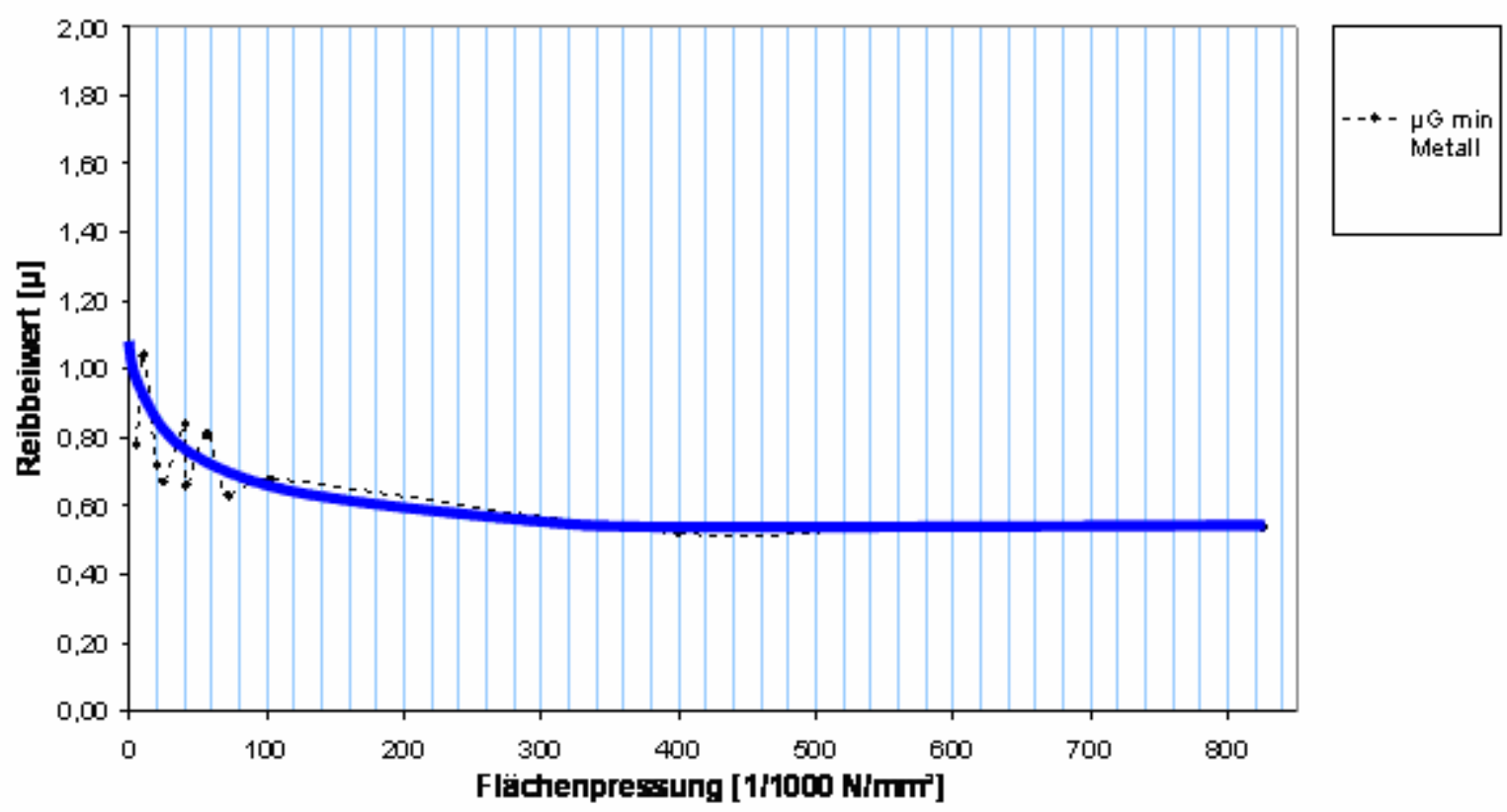

Abbildung 11: Diagramm der minimalen Gleitreibbeiwerte bei unterschiedlichen Flächenpressungen (Metall - ARM - Metall, feucht)

Sofern keine sichtbaren Schäden (z. B. seitliche Einrisse, erkennbare Materialausbrüche oder Fehlstellen) an Antirutschmatten zu erkennen sind, kann davon ausgegangen werden, dass die für die jeweilige Materialpaarung ermittelten Reibbeiwerte wirksam sind. Leichte Verschleißspuren (z. B. angeraute Oberfläche, Schleifspuren) führen nicht zu einer merklichen Reduzierung des Reibbeiwertes. Eine Mehrfachverwendung einer optisch intakten ARM mit geringfügigen Gebrauchsspuren ist aus diesem Grund zulässig.

\section{Notwendigkeit einer seriösen und kompetenten Beratung}

Die komplexen Abhängigkeiten der reibungsrelevanten Einflussfaktoren führen dazu, dass die Anwender, das sind in der Regel die für die Ladungssicherung verantwortlichen Absender oder Verlader und die Lkw-Fahrer, weitgehend überfordert sind, eine für die jeweiligen Aufgaben optimal geeignete ARM auszuwählen. Wichtig und zwingend erforderlich ist vor diesem Hintergrund eine seriöse und kompetente Beratung der Anwender durch die ARM-Anbieter.

Das setzt jedoch auch die Ermittlung geeigneter Kennwerte zur Bewertung der ARM-Qualität (vgl. VDI 2700 Blatt 15) nach geeigneten Prüfrichtlinien und -normen durch zuverlässige Prüfinstitute zwingend voraus.

Generell gilt: „Billig ist nicht preiswert!“ 


\section{Literatur}

[Biermann2005]

[Biermann2001]

[DIN30782-E]

[Strecker2000] [VDI2700]
Biermann, N. (2005): Antirutschmatten für die Ladungssicherung, Deutscher Fachverlag / Schriftenreihe Transport- und Verpackungslogistik Forschungsberichte und Fachbeiträge, Band 65; Hrsg.: Prof. Dr.-Ing. R. Jansen, 2005 Biermann, N. (2001): Ermittlung der Reibbeiwerte von Materialpaarungen im Hinblick auf die Gewährleistung einer anforderungsgerechten Ladungssicherung, Schlussbericht zu AiF-FV-Nr.: 12292 N, 2001.

DIN 30 782-E; Transportbelastungen - Grundlagen und Übersicht über die NormenStruktur, Teil 2: Transportbeanspruchung; Mechanisch-dynamische Beanspruchung; Schwingungen und Stoßbelastungen beim Straßentransport, Beuth Verlag GmbH, Berlin, 1996

Strecker, H. (2000): Verantwortlichkeit des Verladers; in: Gefahrgut 12/2000 VDI 2700 „Ladungssicherung auf Straßenfahrzeugen“, Beuth Verlag GmbH, Berlin, 2004. 